\title{
Thermal response of structured and contaminated carbon surfaces to heat pulses
}

\author{
D. Hildebrandt * and A. Duebner \\ EURATOM Association Max-Planck-Institut für Plasmaphysik, Teilinstitut Greifswald, \\ Wendelsteinstaße 1, 17491 Greifswald, Germany
}

\begin{abstract}
Experiments involving well-defined heat pulses of laser radiation on the CFC target material NB31 and plasma exposed CFC-samples with known composition and thickness of the contamination layer have been performed to investigate the thermal properties and behaviour at the surface. Special attention is focussed on the dependence of the surface temperature enhancement and its temporal evolution on the amount of the deposited material.

The measured surface temperature excursions are compared with values of analytical and numerical solutions of the heat diffusion equation taking into account a surface region with low thermal conductivity or poor thermal contact to the underlying substrate material.
\end{abstract}

JNM Keywords: Carbon, Heat Treatment, Laser, Microstructure, Thermodynamic Properties PSI17 Keywords: Carbon based materials, Divertor tiles, Laser application, Power deposition, IR thermography

PACS codes: $44.50,44.30,44.10$

*Corresponding author: Max-Planck-Institut für Plasmaphysik, Teilinstitut Greifswald, Wendelsteinstaße 1, 17491 Greifswald, Germany e-mail address: dieter.hildebrandt@ipp.mpg.de 


\section{Introduction}

Important aims of the Wendelstein 7-X stellarator experiment presently under construction are the studies of energy and particle exhaust under quasi-stationary conditions and the development of a reactor relevant divertor system. The design of the target plates should keep the local power load below $10 \mathrm{MW} / \mathrm{m}^{2}$. The target elements consists of the carbon fiber composite (CFC) material NB31 connected to water cooled CuCrZr-structures [1]. The temperature of the tile surface exposed to the plasma is expected to rise up to values of 1300 $\mathrm{K}$. To prevent damage of target elements by overheating a continuous supervision of the whole target surface area by thermography is essential.

Such measurements are known to be affected by a reduced thermal conductivity at the surface due to the morphology of the CFC material [2] and surface modification occurring during plasma exposure. Uncertainties in thickness and thermal conductivity of deposited surface layers can lead to faulty interpretation of thermographic measurements $[3,4]$.

The accuracy of temperature measurements on the CFC material NB31 at surface heat loading using IR cameras operating in different wave length regions has been examined with respect to morphological effects for samples of pre-series W7-X target tiles.

The thermal response of contaminated surfaces on heat pulses has been investigated for samples from removed tiles of the Divertor II of the tokamak ASDEX-UPGRADE.

Temperature excursions measured with high spatial (up to $30 \mu \mathrm{m}$ ) and temporal resolution (up to $100 \mu \mathrm{s}$ ) during and after heat pulses by laser radiation are compared with values of the analytical and numerical solution of the heat diffusion equation taking into consideration a surface region with low thermal conductivity or poor thermal contact to the underlying substrate material.

\section{Experimental}

The target tiles for W7-X are manufactured by Plansee Ag [www.plansee.com] using CFCmaterial NB31 produced by Snecma [www.snecma.com]. They are described in detail elsewhere [1] and are investigated before heat load tests using ion beams.

The other samples (CFC material SEPCARB N11) were cut from a tile of the ASDEXUpgrade divertor II. The tile was located at the separatrix position of the inner divertor for five years of operation (1997-2002). Depth profiling using secondary ion mass spectrometry (SIMS) was performed to analyze the contamination layer. It consists mainly of carbon, boron, deuterium and hydrogen with traces of silicon, iron and tungsten. The deposited 
material amount was determined using calibrated sputtering rates for a-C:D layers checked by a Round-Robin experiment [5]. Amounts up to $0.03 \mathrm{~kg} / \mathrm{m}^{2}$ have been measured. With an assumed density $\rho$ of $2 * 10^{3} \mathrm{~kg} / \mathrm{m}^{3}$ this value corresponds to a layer thickness $\mathrm{d}$ of about 15 $\mu \mathrm{m}$. The samples were irradiated by light of wavelength $1.06 \mu \mathrm{m}$ from a pulsed Nd:YAG laser or by light of wavelength $807 \mathrm{~nm}$ from a cw diode laser. The actual absorbed heat flux has been calibrated by calorimetry [2].

The light of the diode laser has been focused to a spot with Gaussian shaped profile and a standard deviation $\sigma$ of $0.5-2 \mathrm{~mm}$. Heat fluxes with central densities $\mathrm{P}_{0}$ up to $12 \mathrm{MW} / \mathrm{m}^{2}$ and durations $\tau$ of up to $1 \mathrm{~s}$ have been applied. The light pulses from the Nd:YAG laser had a duration up to $20 \mathrm{~ms}$. Heat fluxes with central densities up to $110 \mathrm{MW} / \mathrm{m}^{2}$ can be applied at a spot size of $4 \mathrm{~mm}$ (Gaussian or rectangular shaped profile).

The surface temperature excursion during and after the heat pulses has been measured by IRcameras operating in the MIR and FIR wavelength region between 3 and $5 \mu \mathrm{m}$ (InSb detector array with 256x320 pixels) and 8-15 $\mu \mathrm{m}$ (VOx-microbolometer array with 240x320 pixels). The cameras have been calibrated versus black body radiation. The emissivity of the materials has been determined by comparison the measured data with temperature values in drilled holes at uniform sample heating and assuming that the emissivity equals one in the holes. Thermal images have been taken with a spatial resolution of up to $30 \mu \mathrm{m} /$ pixel and frame rates of $315 \mathrm{~Hz}$ or up to $10 \mathrm{kHz}$ at reduced image size $(3-5 \mu \mathrm{m})$ or $50 \mathrm{~Hz}(8-15 \mu \mathrm{m})$.

\section{Modeling}

We use both analytical and numerical methods to describe the temporal evolution of the surface temperature at heat loading. Analytic solutions give a transparent parametric dependence of the variables and can be used to check numerical results.

The analytic solution of the 3D-heat conduction equation derived for the central region of the laser spot hitting the substrate given in [2] is completed by a 1D-surface layer model [3,4] taking into consideration a low thermal conductivity in the layer or poor thermal contact between the contamination layer and the substrate. Assuming a heat diffusion coefficient $\kappa_{1}$ corresponding to the thermal conductivity $\lambda_{1}\left(\kappa_{1}=\lambda_{1} / \rho c_{1}, c_{1}\right.$ the specific heat $)$ within this layer the temporal evolution of the temperature increase at the surface is described by

$$
\Delta T=P_{o} * d / \rho c_{l} \kappa_{l} *\left(\Theta\left(\tau_{p}-t\right) *(1-\exp (-t / \tau))+\Theta\left(t-\tau_{p}\right) * \exp \left(-\left(t-\tau_{p}\right) / \tau\right)\right)
$$


with the time constant of the temperature increase and decrease $\tau=d^{2} / \kappa_{1} . P_{0}$ is the central heat flux density, $\tau_{\mathrm{p}}$ the exposure time and $\Theta(t)$ the Heavyside function.

Alternatively, in the case of poor thermal contact $d / \rho c_{1} \kappa_{1}$ has to be replaced in equation (1) by $1 / \alpha$ with $\alpha$ the heat exchange coefficient between the surface layer and the bulk material. For simplicity the heat conduction coefficient $\lambda$, the heat diffusion coefficient. $\kappa$ and the specific heat capacity $\mathrm{c}$ are assumed to be constant for both the CFC substrate and the layer.

Numerical calculations have been done by a simulated surface layer on top of the bulk material using the finite element code ANSYS [www.ansys.com]. For the calculations the following temperature dependent parameters for the CFC material have been used:

$$
\begin{aligned}
& \lambda_{\mathrm{x}}(\mathrm{T})=400 * \exp (-\mathrm{T} / 315)+105, \lambda_{\mathrm{yz}}(\mathrm{T})=139 * \exp (-\mathrm{T} / 315)+42 \text { for }(\mathrm{NB} 31) \text { or } \\
& \lambda_{\mathrm{x}, \mathrm{Z}}(\mathrm{T})=310 * \exp (-\mathrm{T} / 315)+105, \lambda_{\mathrm{y}}(\mathrm{T})=160 * \exp (-\mathrm{T} / 315)+48 \text { for }(\mathrm{N} 11) \\
& \mathrm{c}(\mathrm{T})=2060 *(1-\exp (-0,00166 * \mathrm{~T})) \text { with } \lambda \text { in } \mathrm{W} / \mathrm{Km} \text { and } \mathrm{c} \text { in } \mathrm{J} / \mathrm{kgK} \text {. }
\end{aligned}
$$

The specific heat of the layer $c_{1}$ and its density are set to $770 \mathrm{~J} / \mathrm{kgK}$ and $2 * 10^{3} \mathrm{~kg} / \mathrm{m}^{3}$, respectively. Its thermal conductivity is an adjustable parameter assumed to be constant.

\section{Morphological effects of NB31 on surface temperature}

Fig. 1 shows in the upper part a thermal image of the CFC material NB31 taken by the InSbcamera with $30 \mu \mathrm{m}$ resolution during application of a heat pulse. It indicates that parts of the fibres have much higher temperature values (emissivity 0.9) than $500 \mathrm{~K}$ predicted by numerical modeling.

The time history of the surface temperature at the centre of a laser spot measured by two cameras operating at different wavelength regions with an ordinary spatial resolution of $1 \mathrm{~mm}$ is shown in fig.3. under a heat pulse with duration of $100 \mathrm{~ms}$.

Numerically calculated results seen in fig. 2 are obtained for the bulk material. A disturbed surface region with reduced thermal conductivity is then added. As demonstrated in fig. 2 the temperature excursion measured by the FIR-camera can be quite well modelled by a $25 \mu \mathrm{m}$ thick surface region with an effective thermal conductivity of $10 \mathrm{~W} / \mathrm{Km}$. The numerically calculated results are within $10 \%$ of the analytic solution obtained by combining eq(1) of this paper and eq(1) of ref [2]. Alternatively, modeling with an effective heat transmission coefficient between the simulated layer and the substrate of $250 \mathrm{~kW} / \mathrm{m}^{2} \mathrm{~K}$ would give similar results. 
Higher temperature values obtained with the MIR-camera in fig.3 are the result of an overestimation due to the variation in temperature across the surface [2].

Because the observed temperature pattern changes with the applied heat flux density the error in MIR-measurements also changes. In the present studies it was found that the overestimation is always smaller than $65 \mathrm{~K}$ for the CFC-material NB31 under applied heat pulses with flux densities up to $100 \mathrm{MW} / \mathrm{m}^{2}$.

\section{Thermal effects on contaminated CFC-material}

The thermal response of contaminated surfaces on heat pulses has been investigated for the plasma-exposed N11-samples from the inner divertor of ASDEX-Upgrade with heat pulses from the diode laser. The IR-data from the centre of the laser spot is taken with frame rates up to $10 \mathrm{kHz}$.

Fig.3a shows the temperature excursions for a sequence of heat pulses. At the beginning of the sequence the surface temperature reaches about $1600 \mathrm{~K}$ and decreases for successive pulses up to level of about $1100 \mathrm{~K}$.

A single square pulse is shown in fig. $3 \mathrm{~b}$ together with calculated results assuming a thermal conductivity of $0.1 \mathrm{~W} / \mathrm{Km}$ for the contamination. The measured cooling time is about $1.5 \mathrm{~ms}$ and agrees well with the value calculated analytically using equation (1). The numerically calculated values are within of $10 \%$ of the analytic solution and the differences consistent with the temperature variation of specific heat and thermal conductivity in the bulk material. Besides the temperature decrease during the pulse series the cooling time also decreases continuously. The last measured temperature excursion in fig.3a has a time constant of only $0.5 \mathrm{~ms}$.

The decreasing temperature level during successive heat pulses seen in fig. 3 indicates changes of the thermal parameters at the surface due to degradation of the contamination. This degradation is also found to start after about $150 \mathrm{~ms}$ when a continuous heat flux of same power density $\left(12 \mathrm{MW} / \mathrm{m}^{2}\right)$ is applied. The removal of the contamination is post-mortem confirmed by a crater produced by the laser spot and the depletion of deuterium inside the crater. Crater depths measured by a laser profilometer were found up to $15 \mu \mathrm{m}$ indicating that the density of the contamination is probably somewhat lower than $2 \mathrm{~kg} / \mathrm{m}^{3}$.

Similar observations of layer decomposition are already observed for a thinner contamination $\left(\rho \mathrm{d}=0.01 \mathrm{~kg} / \mathrm{m}^{2}\right)$ on samples from the inner divertor I of ASDEX-Upgrade when higher power flux densities $\left(28 \mathrm{MW} / \mathrm{m}^{2}\right)$ heated the contamination beyond $1000 \mathrm{~K}$ [2]. The present results 
support the hypothesis that the contamination layer is degraded by thermal desorption and the decisive parameter is obviously the surface temperature itself [2].

Fig.4 shows the dependence of the spot temperature on the original thickness of the contamination for different samples from the divertor II at the beginning and the end of a 12 $\mathrm{MW} / \mathrm{m}^{2}$ heat pulse with duration of $1 \mathrm{~s}$. For comparison numerical calculations were made for two cases. Firstly, the layer has low thermal conductivity $\left(\lambda_{1}=0.1 \mathrm{~W} / \mathrm{Km}\right)$ and good thermal contact to the substrate $\left(\alpha=10^{6} \mathrm{~kW} / \mathrm{Km}^{2}\right)$. In the other case the layer has the same thermal conductivity as the substrate but the thermal contact is poor $\left(\alpha=5 \mathrm{~kW} / \mathrm{Km}^{2}\right)$.

Despite the data scattering and the fact that due to modifications during heating the dependence of the measured spot temperature on the thickness of the contamination layer is somewhat ambiguous, it is quite obvious, that the measured results are much better described by low thermal conductivity.

Disregarding the thermal history at the spot location strong correlation between the temperature increase and the characteristic cooling time is experimentally found (see fig.5).

The relation between the temperature increase and the characteristic cooling time is well reproduced by numerical calculations. The results are also within of $20 \%$ of the analytical solution $\tau=\rho c_{1} \lambda_{1} * \Delta \mathrm{T}^{2} / \mathrm{P}_{0}^{2}$ (eq.2) obtained using eq(1) with $\lambda_{1}=0,1 \mathrm{~W} / \mathrm{Km}$ and $\mathrm{T}=\mathrm{T}_{0}+\Delta \mathrm{T}$. Here uncertainties of the actual contamination thickness are elimated.

It has to be noted that the numerical calculations matches the experimental data better by using constant thermal parameters for the layer rather than values with a relative temperature dependence similar to that of the CFC material.

\section{Conclusions for thermography on W7-X}

Morphological effects at the surface of the CFC-material NB31 are described by a $25 \mu \mathrm{m}$ thick surface region with a reduced thermal conductivity of $10 \mathrm{~W} / \mathrm{Km}$ or alternatively by an effective heat transmission coefficient between the surface region and the substrate of 250 $\mathrm{kW} / \mathrm{m}^{2} \mathrm{~K}$. The mean temperature enhancement caused by this surface region should be only $25 \mathrm{~K}$ at a heat flux density of $10 \mathrm{MW} / \mathrm{m}^{2}$ as expected in $\mathrm{W} 7-\mathrm{X}$. The overestimation of the temperature measured in the MIR region with ordinary resolution of some $\mathrm{mm}$ is expected to be lower than $35 \mathrm{~K}$ due to the non-uniform distribution (calculated from data of fig.2).

Composition and thermal properties of the deposited material on the divertor targets of W7-X are expected to be similar to that found on the target tiles of ASDEX-Upgrade. Modeling 
matches the measured results with a thermal conductivity of the deposited material of 0.1 $\mathrm{W} / \mathrm{Km}$. The partial removal of this contamination observed under surface heat loading of 12 $\mathrm{MW} / \mathrm{m}^{2}$ is encouraging. This would allow temperature supervising of components with a thermal response time larger than $1 \mathrm{sec}$ and critical surface temperature of about $1300 \mathrm{~K}$ as in the case of the target plates of W7-X at least for contamination amounts up to $0.02 \mathrm{~kg} / \mathrm{m}^{2}$.

However, the surface temperature level is still somewhat higher for original contamination amounts larger than $0.02 \mathrm{~kg} / \mathrm{m}^{2}$ after $1 \mathrm{sec}$ exposure of critical heat load. The questions remain: What happens when much larger amounts are deposited during quasistationary operation? Do temperature levels higher than $1300 \mathrm{~K}$ in W7-X indicate thick contamination or heat flux densities higher than $12 \mathrm{MW} / \mathrm{m}^{2}$ in this case? Temperature relaxation measurements with high temporal resolution $(<0.5 \mathrm{~ms})$ after short heat pulses can be able to reduce the uncertainties. 


\section{References}

[1] J. Boscary et al., Fus. Eng. And Design 75-79 (2005) 451

[2] D. Hildebrandt et al., J. Nucl. Mater.337-339 (2005), 1064

[3] S. Clement et al., J. Nucl. Mater. 266-269(1999) 285

[4] P. Andrew et al., J. Nucl. Mater.337-339(2005), 99

[5] R. Behrisch et al., J. Nucl. Mater. 281(200) 42 


\section{Figure Captions:}

Fig.1: Thermal image of NB31 taken by the InSb camera with a spatial resolution of 30 $\mu \mathrm{m} /$ pixel during a laser pulse; applied heat pulse $\mathrm{P}_{0}=30 \mathrm{MW} / \mathrm{m}^{2}, \tau_{\mathrm{p}}=7 \mathrm{~ms}$, diameter $8 \mathrm{~mm}$; The temperature varies between $450 \mathrm{~K}$ (dark regions) and $800 \mathrm{~K}$ (bright regions).

Fig.2: Comparison of the surface temperature excursion measured on the NB 31 material in the MIR and FIR region with numerically calculated results for the bulk material (dashed line) and an added assumed $25 \mu \mathrm{m}$ surface region of low thermal conductivity (solid line); applied heat pulse $\mathrm{P}_{0}=2 \mathrm{MW} / \mathrm{m}^{2}, \tau_{\mathrm{p}}=100 \mathrm{~ms}, \sigma=1,6 \mathrm{~mm}$.

Fig.3 (a)-top: Temperature excursions for a sequence of heat pulses with duration of $50 \mathrm{~ms}$ for a contamination of $0.02 \mathrm{~kg} / \mathrm{m}^{2}$; applied heat pulses $\mathrm{P}_{0}=12 \mathrm{MW} / \mathrm{m}^{2}, \sigma=0.56 \mathrm{~mm}$.

(b)-bottom: Detail of the upper figure together with calculated results

Fig.4: Dependence of the measured spot temperature on the original deposited material amount at the beginning and the end of a $12 \mathrm{MW} / \mathrm{m}^{2}$ heat pulse with duration of $1 \mathrm{~s}$. Numerical calculations were made for low thermal conductivity of $\lambda_{1}=0.1 \mathrm{~W} / \mathrm{Km}$ (solid line) and for poor thermal contact with $\alpha=5 \mathrm{~kW} / \mathrm{Km}^{2}$ (dashed line).

Fig.5: Correlation between the measured spot temperature and the characteristic cooling time; the result of the numerical calculations (solid line) is obtained using $\lambda_{1}=0.1 \mathrm{~W} / \mathrm{Km}$. The symbols represent different samples. 


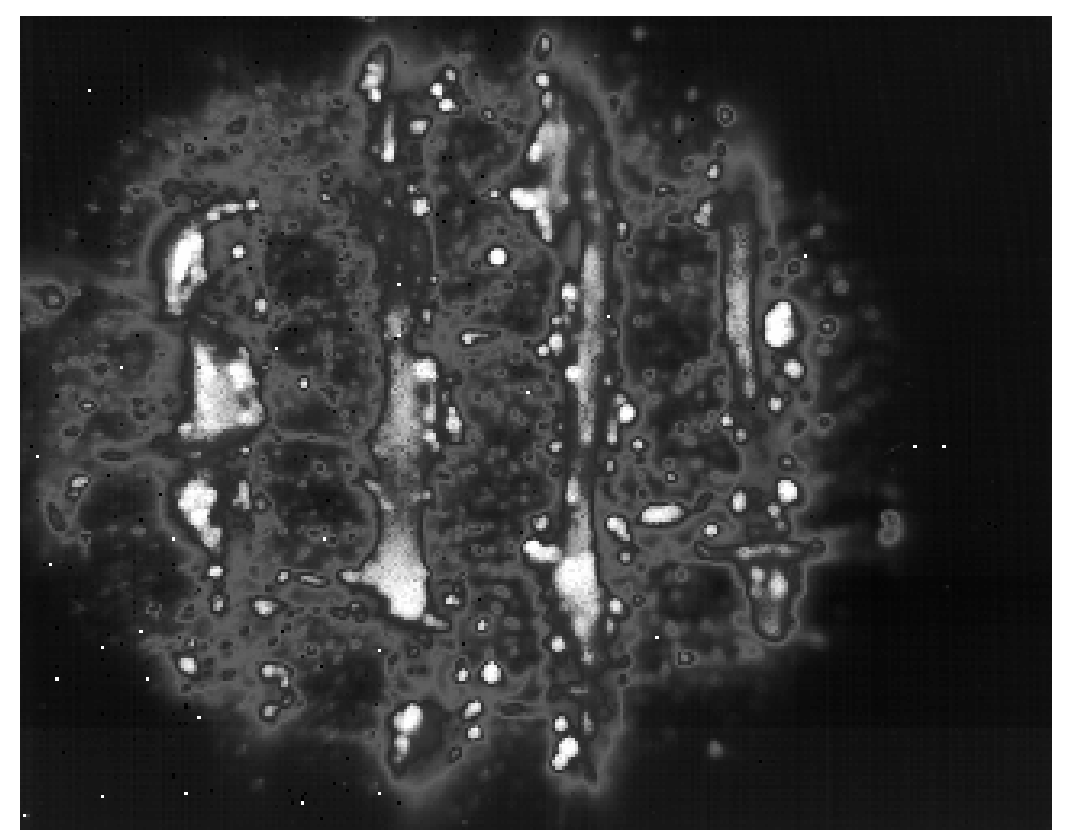

Fig.1 


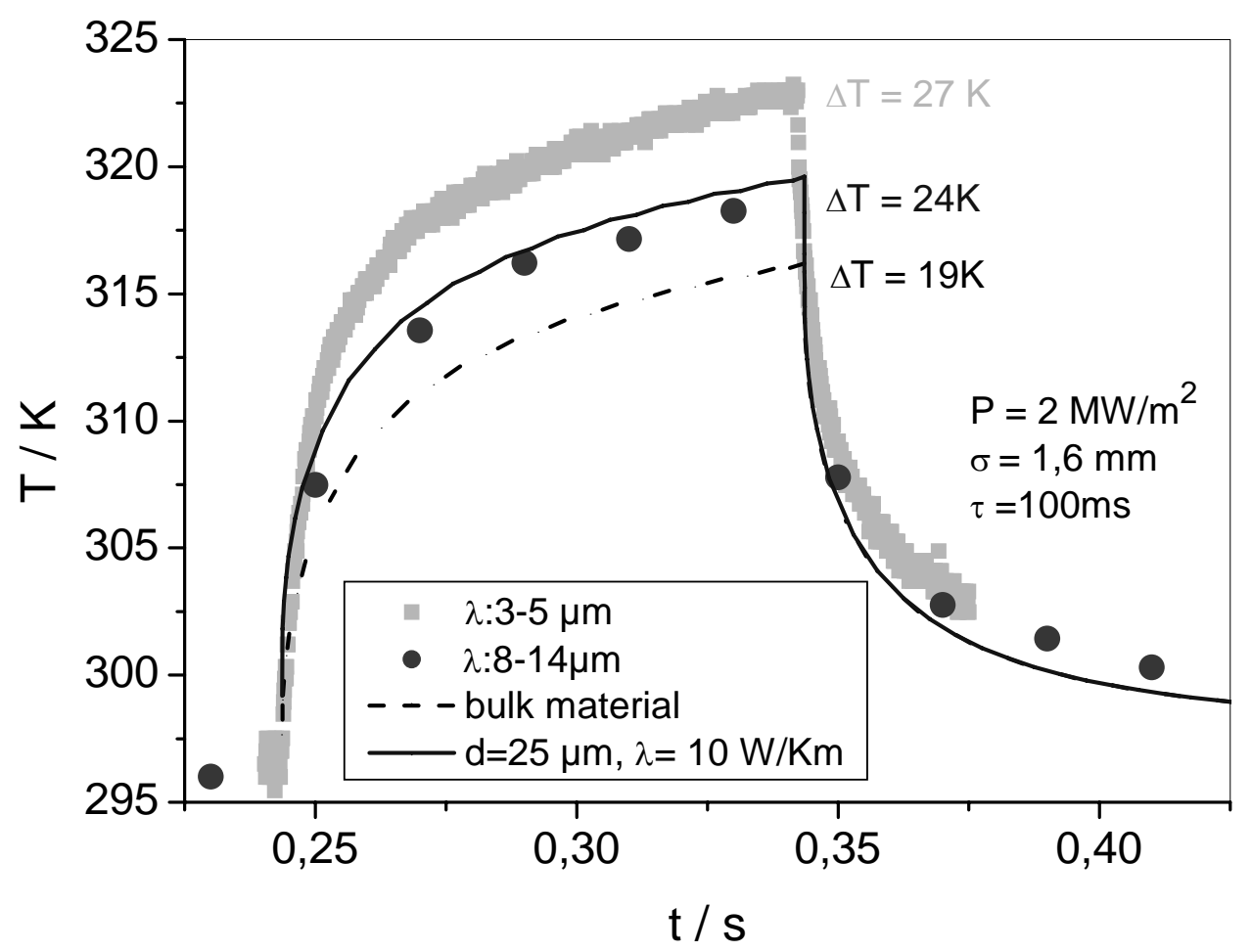

Fig. 2 

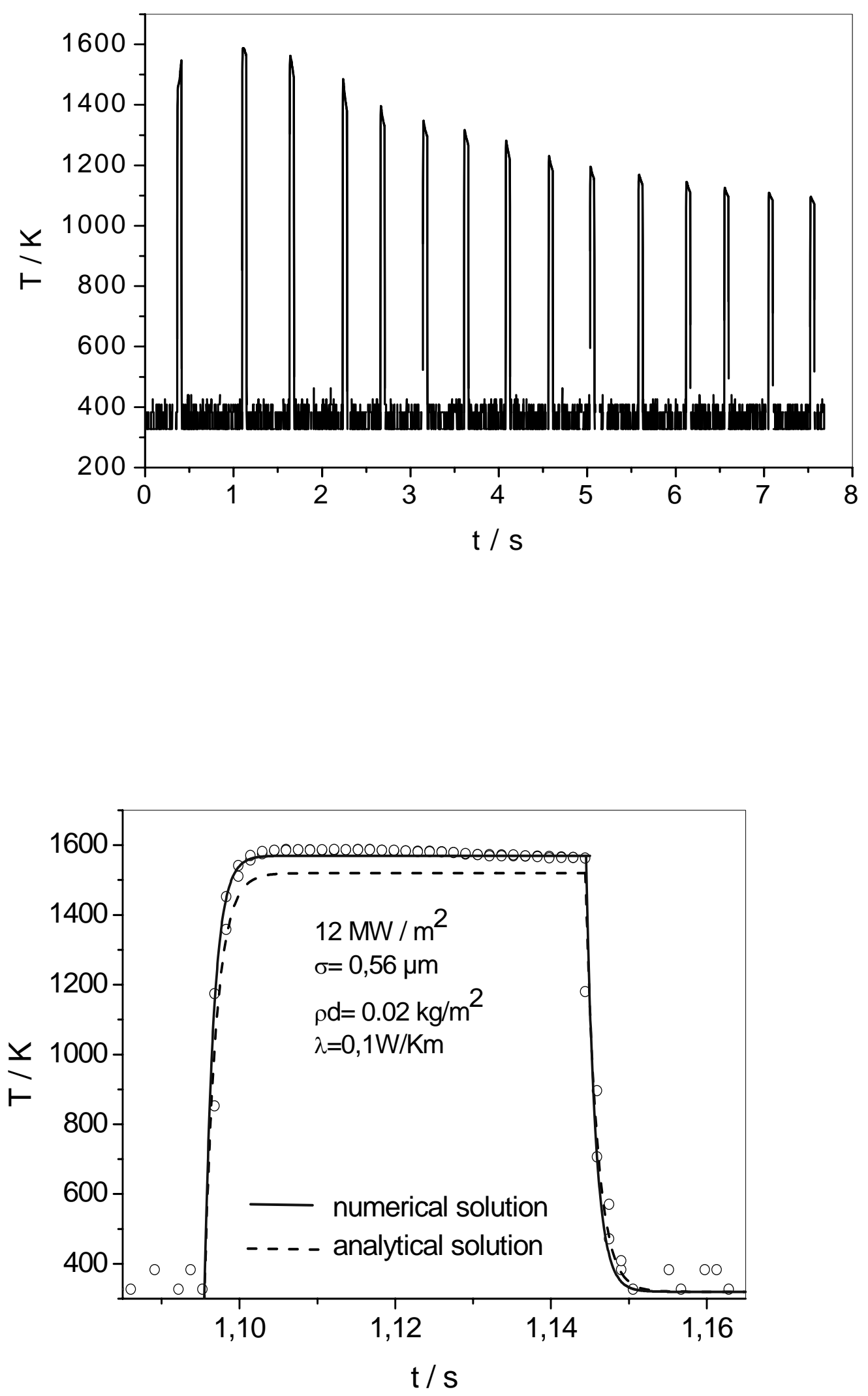

Fig.3a and $\mathrm{b}$ 


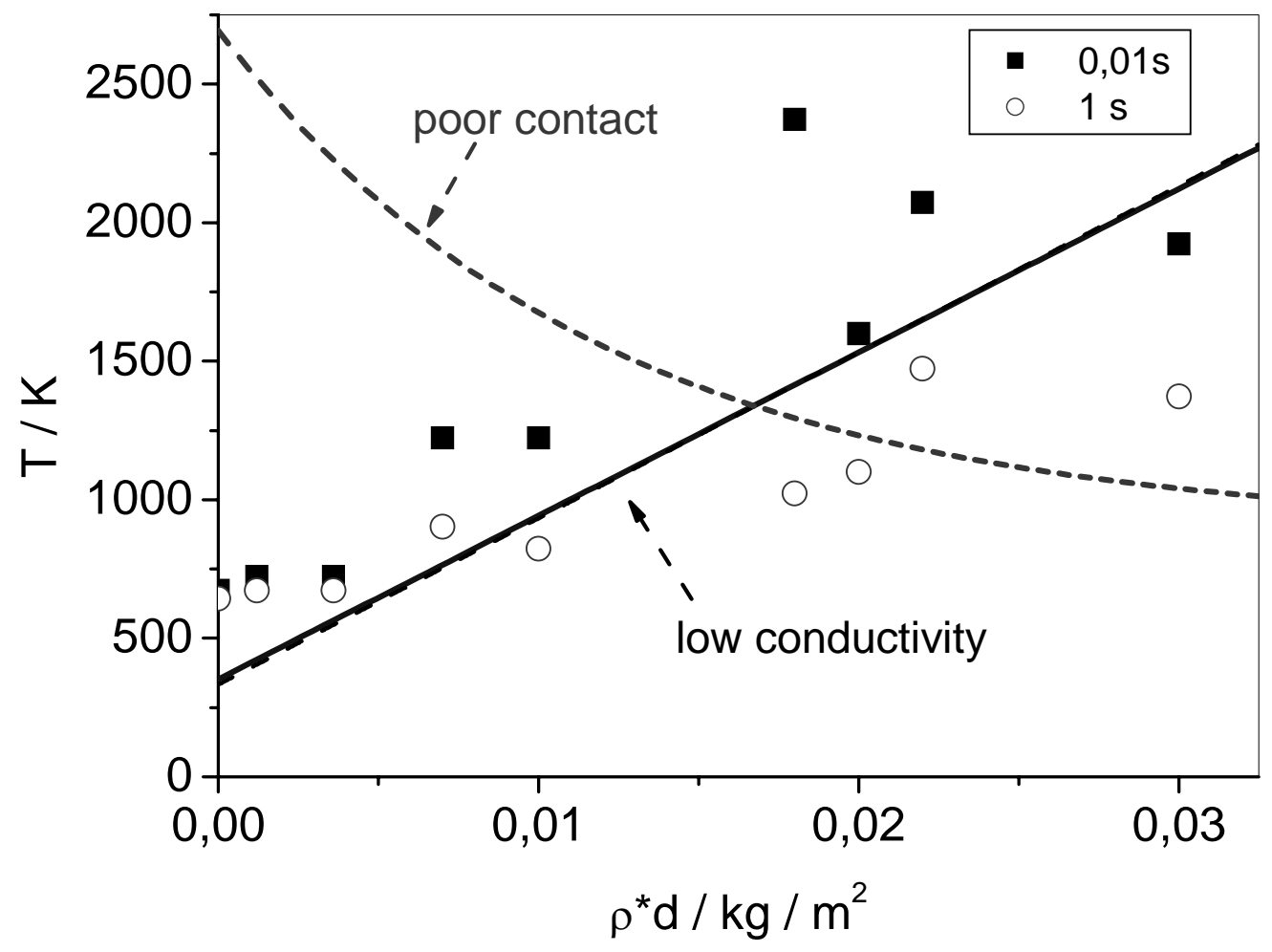

Fig.4 


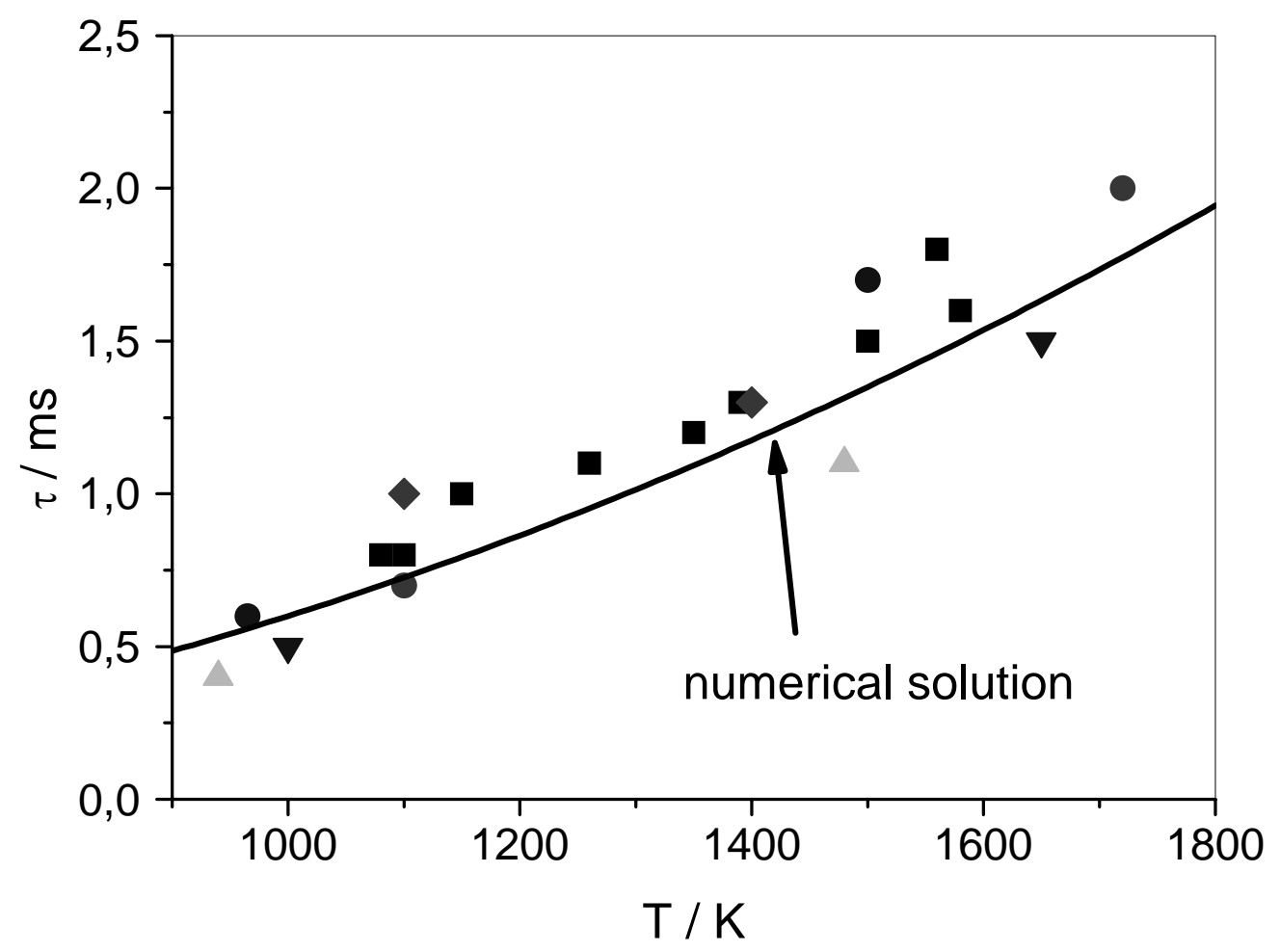

Fig. 5 